\title{
Synergistic effects of histologic subtype, T-stage, and M-stage in the prognosis of differentiated thyroid cancer: a retrospective observational study
}

\author{
Ling Zhou ${ }^{1 \#}$, Shipei Wang ${ }^{1 \#}$, Sichao Chen ${ }^{1}$, Yihui Huang ${ }^{1}$, Di Hu ${ }^{1}$, Wei Wei ${ }^{3}$, Chao Zhang ${ }^{4}$, Min Wang ${ }^{1}$, \\ Wei Zhou ${ }^{1}$, Wen Zeng ${ }^{2}$, Zeming Liu ${ }^{1}$, Liang Guo ${ }^{1}$ \\ ${ }^{1}$ Department of Plastic Surgery, ${ }^{2}$ Department of Ophthalmology, Zhongnan Hospital, Wuhan University, Wuhan, China; ${ }^{3}$ Department of Pediatrics, \\ St John Hospital and Medical Center, Detroit, MI, USA; ${ }^{4}$ Department of Cardiovascular Surgery, Union Hospital, Tongji Medical College, \\ Huazhong University of Science and Technology, Wuhan, China \\ Contributions: (I) Conception and design: S Wang, L Zhou; (II) Administrative support: W Zhou, M Wang; (III) Provision of study materials or \\ patients: Z Liu, L Guo; (IV) Collection and assembly of data: W Wei, D Hu, C Zhang; (V) Data analysis and interpretation: S Chen, W Zeng, Y \\ Huang; (VI) Manuscript writing: All authors; (VII) Final approval of manuscript: All authors. \\ \#These authors contributed equally to this work. \\ Correspondence to: Liang Guo; Zeming Liu. Department of Plastic Surgery, Zhongnan Hospital of Wuhan University, Donghu Load 169, Wuchang \\ District, Wuhan, China. Email: guoliangwhzn@163.com; 6myt@163.com.
}

Background: The incidence of differentiated thyroid cancer has increased in many countries during the past few decades. This study aimed to investigate the synergistic effect of clinicopathological factors, including histologic subtype, T stage, and $M$ stage, on the prognosis of differentiated thyroid cancer (DTC). Methods: We collected data on 86,302 patients with DTC from 2004 to 2013 from the SEER database. We extracted multiple variables from the selected object of study. Demographic characteristics consisted of age at diagnosis, sex, year of diagnosis, and race. Pathological characteristics included T stage, $\mathrm{N}$ stage, $M$ stage, multifocality, histologic subtype and extrathyroidal extension. Treatment characteristics included radiation therapy and surgery. Univariate and multivariate analyses were conducted to evaluate the correlation between clinicopathological factors and prognosis. The relative excess risk of synergistic effect (RERI), attributable proportion (AP) of synergistic effect, and synergy index (SI) were used to explore the synergistic effect of these factors on prognosis.

Results: Histologic subtype, T-stage, and M-stage were found to be risk factors for cancer-specific survival and all-cause survival in multivariate analysis. The cancer-specific mortality (CSM) rates per 1,000 person-years for patients were found to be higher in follicular thyroid carcinoma (FTC) patients and patients with T3-T4, M1 status disease. In addition, CSM and all-cause mortality (ACM) were also associated with age, sex, race, $\mathrm{N}$ - stage, extension, radiation treatment, and surgical approach (all, $\mathrm{P}<0.001$ ). We also found that histologic subtype had a synergistic effect with both $\mathrm{T}$ and $\mathrm{M}$ status stage on the prognosis (RERI $=7.431, \mathrm{AP}=0.278, \mathrm{SI}=1.407$; RERI $=37.889, \mathrm{AP}=0.430, \mathrm{SI}=1.771$, respectively). Synergy was also noted between $\mathrm{T}$ - stage and $\mathrm{M}$ - stage (RERI $=134.125, \mathrm{AP}=0.537, \mathrm{SI}=2.168)$.

Conclusions: Histologic subtype, T-stage, and M-stage in different combinations were risk factors for poor prognosis in DTC, and had synergistic effects.

Keywords: Differentiated thyroid cancer; death; synergistic effect; SEER

Submitted Jan 09, 2020. Accepted for publication Jul 08, 2020.

doi: $10.21037 /$ tcr-20-359

View this article at: http://dx.doi.org/10.21037/tcr-20-359 


\section{Introduction}

The incidence of thyroid cancer, the most common malignant tumor of the endocrine system, has increased considerably among both men and women in many countries during the past few decades (1-3). Globally, 567,000 people suffer from thyroid cancer, making it the ninth most frequently diagnosed cancer (1).

Differentiated thyroid cancer (DTC) comprises over $85 \%$ of all thyroid cancers (4), and is seen with increasing incidence in clinical practice (5). Several studies have confirmed the increasing incidence of differentiated thyroid cancer in the USA, Europe, Canada, Brazil, Australia and Saudi Arabia (6). DTC originates from epithelial follicular cell and includes two histological types, namely, papillary thyroid carcinoma (PTC) and follicular thyroid carcinoma (FTC). PTC and FTC account for approximately $80-85 \%$ and $10-15 \%$, respectively, of all thyroid malignancies $(7,8)$. Despite the rising incidence, DTC bears an excellent prognosis with 10 -year overall survival (OS) rates for patients with PTC and FTC of $93 \%$ and $85 \%$, respectively (9).

The etiology and pathogenesis of thyroid cancer remain unclear. Many previous studies have identified various clinicopathological predictors of DTC and designed risk component stratification or staging systems. Several clinical factors, which are considered to be prognostically significant, are included in these systems (10). However, the synergistic effects of the different clinicopathological risk factors have not been evaluated. In this study, we analyzed the synergistic effects of three common clinicopathological characteristics (histologic subtype, $\mathrm{T}$ stage, and $\mathrm{M}$ stage) to determine their relationship to prognosis in patients with DTC. We present the following article in accordance with the STROBE reporting checklist (available at http://dx.doi. org/10.21037/tcr-20-359).

\section{Methods}

\section{Data collection}

The population-based data for this study were extracted from the SEER database established by the National Cancer Institute. Data from patients in the SEER database with a diagnosis of DTC from 2004 to 2013 were included. The research was conducted in accordance with the Declaration of Helsinki (as revised in 2013). Since SEER is a publicly available database with anonymized data, and our study is a retrospective study, no ethical review and informed consent were required.

We put no restriction on age or race. The exclusion criteria were as follows: patients diagnosed with DTC at death or autopsy; primary malignant tumors in other organs; benign or borderline tumors; unknown age, cause of death, or survival time; unavailable or incomplete information on surgery or radiation therapy; and inactive follow-up. Finally, 86,032 patients were included (Figure 1).

We extracted multiple variables from the selected object of study. Demographic characteristics consisted of age at diagnosis ( $<55$ or $\geq 55$ years), sex (male or female), year of diagnosis (2004-2008 or 2009-2013), and race (white, black, or other). Pathological characteristics included T-stage (T1, T2, T3, or T4), N stage (N0 or N1 stage), M stage (M0 or M1 stage), multifocality, histologic subtype (PTC or FTC), and extrathyroidal extension. Treatment characteristics included radiation therapy (none, or refused, radiation beam or radioactive implants, radioisotopes or radiation beam plus isotopes or implants) and surgery (none, lobectomy, subtotal or nearly total thyroidectomy, and total thyroidectomy).

\section{Statistical analysis}

Univariate and multivariate analyses were performed, respectively, using the Chi squared test and binary logistic regression to explore factors associated with prognosis. In order to evaluate the effects of different histologic subtypes, $\mathrm{T}$ stage, and $\mathrm{M}$ stage on cancer-specific mortality (CSM) and all-cause mortality (ACM), we used Cox proportional hazard regression analysis to estimate the hazard ratio (HR) and $95 \%$ confidence interval (CI). The relative excess risk (RERI), attributable proportion (AP), and synergy index (SI) were utilized to assess the interactive effect of these factors on prognosis in DTC patients. When there is no additive synergistic effect, the $95 \%$ CIs of RERI and AP include 0, and the SI includes 1 (11). In other words, a result of RERI $>0, \mathrm{AP}>0$, or $\mathrm{S}>1$ is considered as verifying the existence of a synergistic effect. The Kaplan-Meier method was used to draw survival curves, with log-rank test to determine statistical differences across groups.

All statistical analyses and charts of survival probabilities were performed using the SPSS 22.0 (IBM Corporation, Armonk, NY, USA), Stata/SE version 12 (Stata Corp., College Station, TX, USA), R statistical software (R Core Development Team, Vienna, Austria), and GraphPad Prism version 6 (GraphPad Software Inc., La Jolla, CA). All the statistical tests were two-sided, and statistical significance 


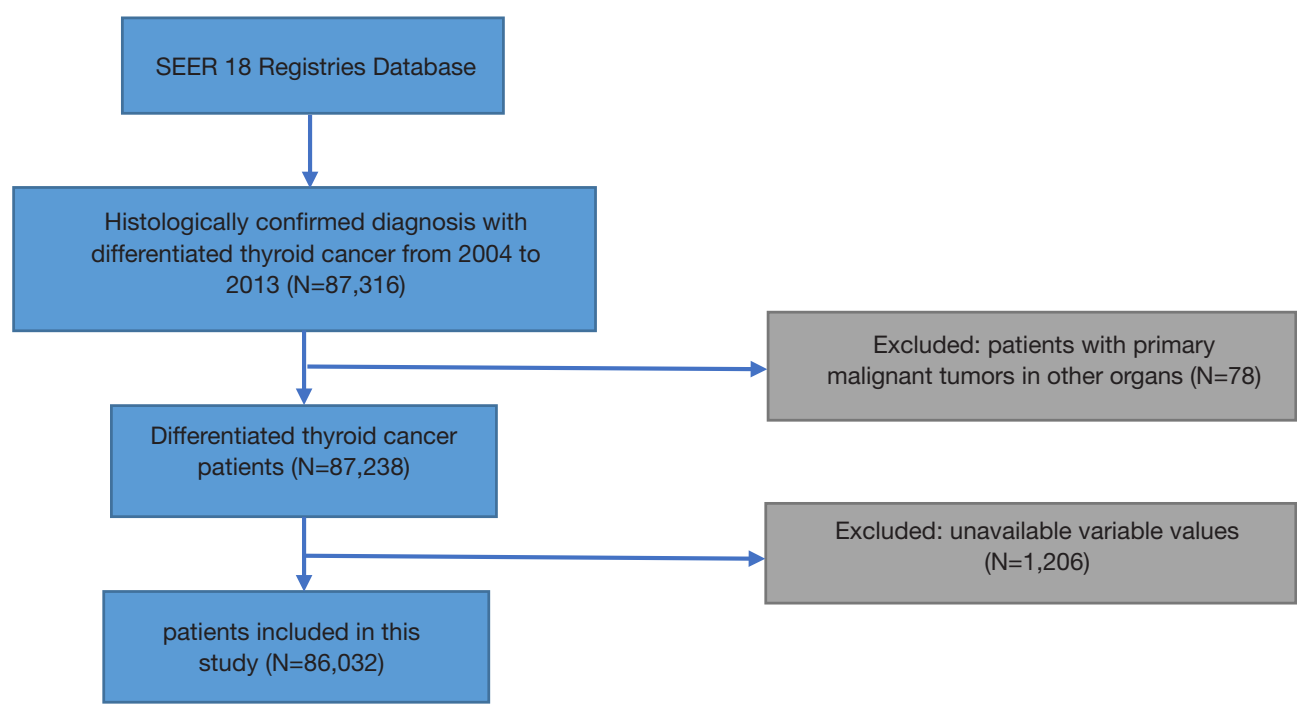

Figure 1 Flowchart of patients identified in this study. SEER, Surveillance Epidemiology and End Results.

was defined as $\mathrm{P}$ value $<0.05$.

\section{Results}

\section{General characteristics of the study population}

The general clinicopathological characteristics of the 86,032 patients analyzed in this study are shown in Table 1 . There were 4,810 (5.6\%) patients with FTC, 52,049 (60.5\%) patients with T1 stage, 18,628 (21.7\%) patients with LNM, and $874(1.0 \%)$ patients with distant metastasis.

\section{CSM and ACM rates per 1,000 person-years}

During the follow-up period, cancer-specific death occurred in 579 patients in the PTC group and 96 patients in the FTC group. The CSM rates per 1,000 person-years were 1.704 (95\% CI: $1.569-1.850)$ for PTC and 4.247 (95\% CI: 3.459-5.216) for FTC (Table 2). In addition, during the follow-up period, all-cause death occurred in 3,064 patients in the PTC group and 323 patients in the FTC group. The ACM rates per 1,000 person-years were 9.057 (95\% CI: 8.739-9.386) for PTC and 14.656 (95\% CI: 13.121-16.370) for FTC (Table 2).

The CSM rates per 1,000 person-years for patients with T1, T2, T3, and T4 DTC were 0.339 (95\% CI: 0.269-0.427), 0.901 (95\% CI: 0.695-1.168), 2.955 (95\% CI: 2.568-3.401), and 28.173 (95\% CI: 25.308-31.362), respectively (Table 2). The CSM rates per 1,000 personyears for patients with M0 and M1 disease were 1.318 (95\%
CI: $1.203-1.444)$ and 66.552 (95\% CI: 57.837-76.581), respectively (Table 2).

During the follow-up period, the ACM rates per 1,000 person-years for patients with T1, T2, T3, and T4 disease were 7.207 (95\% CI: 6.855-7.577), 7.443 (95\% CI: 6.8008.146), 11.503 (95\% CI: 10.713-12.351), and 47.321 (95\% CI: 43.563-51.403), respectively (Table 2). Moreover, the ACM rates per 1,000 person-years for patients with $\mathrm{M} 0$ and M1 disease were 8.648 (95\% CI: 8.346-8.961) and 99.316 (95\% CI: 88.537-111.409), respectively (Table 2). Analysis of the CSM and ACM rates per 1,000 person-years using a combination of histologic subtype, T-stage, and M-stage, yielded similar results (Table 3).

\section{Risk factors for cancer-specific and ACM}

The univariate Cox regression analysis showed that CSM and ACM were related to age, sex, race, histologic type, TNM stage, extension, radiation therapy, and surgical approach. The multivariate Cox regression model revealed that FTC, compared to PTC, was an independent risk factor for both CSM and ACM (Tables S1,S2).

\section{Synergic effects of T stage, LNM, and DM on the prognosis of DTC}

As shown in Table 4, the probability of death in DTC patients with FTC and T3-T4 disease was the highest (HR $=6.719,95 \%$ CI: 4.697-9.611, $\mathrm{P}<0.001)$ when compared 
Table 1 Demographic and clinicopathological characteristics of 86,032 patients with DTC

\begin{tabular}{|c|c|}
\hline Characteristics & Number (\%) \\
\hline \multicolumn{2}{|l|}{ Age at diagnosis (year) } \\
\hline Mean [range] & $49.14[2-105]$ \\
\hline$<55$ years & $54,785(63.7)$ \\
\hline$\geq 55$ years & 31,247 (36.3) \\
\hline \multicolumn{2}{|l|}{ Year of diagnosis } \\
\hline 2004-2008 & 35,389 (41.1) \\
\hline 2009-2013 & $50,643(58.9)$ \\
\hline \multicolumn{2}{|l|}{ Sex } \\
\hline Female & $66,537(77.3)$ \\
\hline Male & $19,495(22.7)$ \\
\hline \multicolumn{2}{|l|}{ Race } \\
\hline White & $70,568(82.0)$ \\
\hline Black & $5,399(6.3)$ \\
\hline Other & $9,086(10.6)$ \\
\hline \multicolumn{2}{|l|}{ T stage } \\
\hline $\mathrm{T} 1$ & $52,049(60.5)$ \\
\hline $\mathrm{T} 2$ & 14,705 (17.1) \\
\hline T3 & $16,394(19.1)$ \\
\hline $\mathrm{T} 4$ & $2,884(3.4)$ \\
\hline Lymph node metastasis & $18,628(21.7)$ \\
\hline Distant metastasis & $874(1.0)$ \\
\hline Multifocality & $34,734(40.4)$ \\
\hline \multicolumn{2}{|l|}{ Histology subtype } \\
\hline PTC & $81,222(94.4)$ \\
\hline FTC & $4,810(5.6)$ \\
\hline Extrathyroidal extension & $13,673(15.9)$ \\
\hline \multicolumn{2}{|l|}{ Radiation therapy } \\
\hline None or refused & $42,209(49.1)$ \\
\hline Radiation beam or radioactive implants & $1,493(1.7)$ \\
\hline $\begin{array}{l}\text { Radioisotopes or radiation beam plus } \\
\text { isotopes or implants }\end{array}$ & $42,330(49.2)$ \\
\hline \multicolumn{2}{|l|}{ Surgery } \\
\hline Lobectomy & $12,289(14.3)$ \\
\hline Subtotal or near-total thyroidectomy & $3,187(3.7)$ \\
\hline Total thyroidectomy & $70,556(82.0)$ \\
\hline
\end{tabular}

DTC, differentiated thyroid cancer; PTC, papillary thyroid cancer; FTC, follicular thyroid cancer. with those with other combinations of histologic subtype and $\mathrm{T}$ stage, after adjustment for age at diagnosis, year at diagnosis, sex, race, $\mathrm{N}$ stage, $\mathrm{M}$ stage, multifocality, extrathyroidal extension, radiation, surgery. In addition, DTC patients with PTC and T3-T4 stage, or FTC and T1-T2 stage are more likely to die $(\mathrm{HR}=4.065,95 \% \mathrm{CI}$ : 2.907-5.684, $\mathrm{P}<0.001$; HR $=3.221,95 \%$ CI: $2.045-5.075$, $\mathrm{P}<0.001$ ), when compared with those of PTC with T1-T2 stage after the adjustment for cofounders, which are also listed in Table 4.

For these results, the RERI was 7.431 (95\% CI: 0.65314.208), which indicates that there would be a 7.431 -fold relative excess risk of death contributed by the additive synergistic effect of FTC histologic subtype and T stage. The AP was 0.278 (95\% CI: 0.088-0.468), which suggests that $27.8 \%$ of deaths with the two risk factors were caused by the synergistic effect. In addition, the SI value (1.407, 95\% CI: 1.069-1.851) was larger than 1, which signifies the existence of a synergistic effect between histologic subtype and $T$ stage on death.

The risk of death in FTC patients with distant metastasis was the highest (HR $=14.163$, 95\% CI: 10.418-19.254) when compared with those with other combinations of histologic subtype and $M$ stage after adjustment for age at diagnosis, year of diagnosis, sex, race, T stage, $\mathrm{N}$ stage, multifocality, extrathyroidal extension, radiation, and surgery (Table 5). The risk of death was greater in PTC patients with M1 stage or FTC patients with M0 stage (HR $=6.009,95 \%$ CI: 4.904-7.364, $\mathrm{P}<0.001$; HR $=1.590,95 \%$ CI: 1.146-2.205, $\mathrm{P}<0.001)$ than in PTC patients without metastasis. Moreover, there would be a 37.889-fold relative excess risk of death contributed by the additive synergistic effect of histologic subtype and $M$ stage. In addition, $43.0 \%$ of deaths with the two risk factors were caused by the additive synergistic effect. The SI was 1.771 (95\% CI: $1.205-2.603)$.

We also evaluated the synergistic effect between $T$ stage and $\mathrm{M}$ stage on death (Table 6). The risk of death was greatest in DTC patients with T1-T2 stage and M1 stage (HR $=39.055$, 95\% CI: 26.034-58.588) when compared with those with other combinations of $T$ stage and $M$ stage after adjustment for age at diagnosis, year of diagnosis, sex, race, $\mathrm{N}$ stage, multifocality, histologic subtype, extrathyroidal extension, radiation, and surgery. The risk of death was greater in DTC patients with T3-T4 and M1 or M0 stage $(\mathrm{HR}=32.505,95 \% \mathrm{CI}: 22.260-47.465, \mathrm{P}<0.001$; HR $=4.756,95 \%$ CI: $3.409-6.636, \mathrm{P}<0.001$, respectively) than in patients in the T1-T2 and M0 stage group. According to 
Table 2 Hazard ratios of some factors for the cancer specific deaths and all cause deaths of thyroid cancer

\begin{tabular}{|c|c|c|c|c|c|c|c|c|}
\hline Variable & \multicolumn{4}{|c|}{ Cancer-specific mortality } & \multicolumn{4}{|c|}{ All-cause mortality } \\
\hline \multicolumn{9}{|c|}{ Histology subtype } \\
\hline PTC & 579 & 0.7 & 1.704 & $1.569-1.850$ & 3,064 & 3.8 & 9.057 & $8.739-9.386$ \\
\hline FTC & 96 & 2.0 & 4.247 & $3.459-5.216$ & 323 & 6.7 & 14.656 & $13.121-16.370$ \\
\hline $\mathrm{T} 1$ & 74 & 0.1 & 0.339 & $0.269-0.427$ & 1,572 & 3.0 & 7.207 & $6.855-7.577$ \\
\hline $\mathrm{T} 2$ & 58 & 0.4 & 0.901 & $0.695-1.168$ & 475 & 3.2 & 7.443 & $6.800-8.146$ \\
\hline T3 & 199 & 1.2 & 2.955 & $2.568-3.401$ & 767 & 4.7 & 11.503 & $10.713-12.351$ \\
\hline $\mathrm{T} 4$ & 344 & 11.9 & 28.173 & $25.308-31.362$ & 573 & 19.9 & 47.321 & $43.563-51.403$ \\
\hline M1 & 200 & 22.9 & 66.552 & $57.837-76.581$ & 296 & 33.9 & 99.316 & $88.537-111.409$ \\
\hline
\end{tabular}

DTC, differentiated thyroid cancer; PTC, papillary thyroid cancer; FTC, follicular thyroid cancer.

Table 3 Hazard ratios of some combined factors for the cancer specific deaths and all cause deaths of thyroid cancer

\begin{tabular}{|c|c|c|c|c|c|c|c|c|}
\hline Variable & \multicolumn{4}{|c|}{ Cancer-specific mortality } & \multicolumn{4}{|c|}{ All-cause mortality } \\
\hline PTC and T1-T2 stage & 109 & 0.2 & 0.405 & $0.335-0.490$ & 1,908 & 3.0 & 7.139 & $6.823-7.471$ \\
\hline PTC and T3-T4 stage & 470 & 2.7 & 6.513 & $5.945-7.135$ & 1,156 & 6.6 & 16.148 & $15.238-17.112$ \\
\hline FTC and T3-T4 stage & 73 & 4.4 & 9.638 & $7.599-12.224$ & 184 & 11.0 & 25.088 & $21.651-29.070$ \\
\hline \multicolumn{9}{|l|}{ Histology subtype and M stage } \\
\hline PTC and MO stage & 429 & 0.5 & 1.271 & $1.155-1.398$ & 2,830 & 3.5 & 8.424 & $8.116-8.743$ \\
\hline \multicolumn{9}{|l|}{$\mathrm{T}$ stage and $\mathrm{M}$ stage } \\
\hline $\mathrm{T} 1-\mathrm{T} 2$ stage and $\mathrm{M} 0$ stage & 98 & 0.1 & 0.346 & $0.283-0.423$ & 1,988 & 3.0 & 7.076 & $6.768-7.398$ \\
\hline T1-T2 stage and M1 stage & 34 & 13.2 & 33.855 & $24.190-47.381$ & 59 & 22.9 & 58.749 & $45.518-75.825$ \\
\hline T3-T4 stage and M0 stage & 377 & 2.0 & 4.848 & $4.377-5.369$ & 1,103 & 5.9 & 14.333 & $13.506-15.211$ \\
\hline T3-T4 stage and M1 stage & 166 & 26.9 & 83.604 & $71.638-97.569$ & 237 & 38.5 & 120.473 & $105.926-137.016$ \\
\hline
\end{tabular}

DTC, differentiated thyroid cancer; PTC, papillary thyroid cancer; FTC, follicular thyroid cancer. 
Table 4 Measures for estimation of synergic effect between histology subtype and T stage for the cancer specific survival of DTC

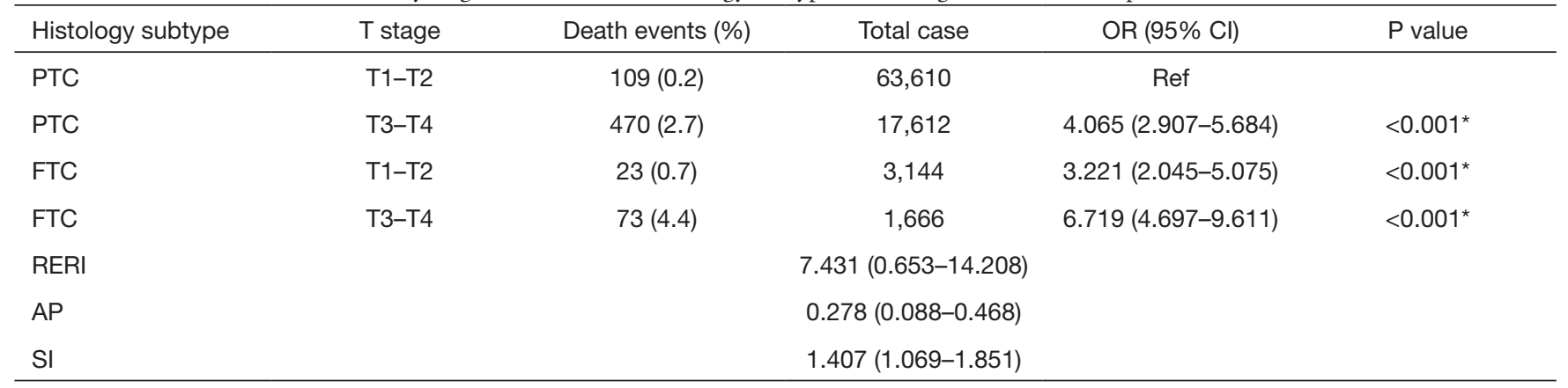

Adjusted for age at diagnosis, year at diagnosis, sex, race, $\mathrm{N}$ stage, M stage, multifocality, extrathyroidal extension, radiation, surgery. *, represent the P value $<0.05$. DTC, differentiated thyroid cancer; PTC, papillary thyroid cancer; FTC, follicular thyroid cancer; RERI, relative excess risk; AP, attributable proportion; SI, synergy index.

Table 5 Measures for estimation of synergic effect between histology subtype and $M$ stage for the cancer specific survival of DTC

\begin{tabular}{|c|c|c|c|c|c|}
\hline Histology subtype & M stage & Death events (\%) & Total case & $\mathrm{HR}(95 \% \mathrm{Cl})$ & $P$ value \\
\hline PTC & M1 & $150(20.9)$ & 718 & 6.009 (4.904-7.364) & $<0.001^{*}$ \\
\hline FTC & MO & $46(1.0)$ & 4,654 & $1.590(1.146-2.205)$ & $<0.001^{*}$ \\
\hline FTC & M1 & $50(32.1)$ & 156 & $14.163(10.418-19.254)$ & $<0.001^{*}$ \\
\hline $\mathrm{AP}$ & \multicolumn{5}{|c|}{$0.430(0.214-0.647)$} \\
\hline SI & \multicolumn{5}{|c|}{$1.771(1.205-2.603)$} \\
\hline
\end{tabular}

Adjusted for age at diagnosis, year at diagnosis, sex, race, T stage, $\mathrm{N}$ stage, multifocality, extrathyroidal extension, radiation, surgery. *, represent the $\mathrm{P}$ value $<0.05$. DTC, differentiated thyroid cancer; PTC, papillary thyroid cancer; FTC, follicular thyroid cancer; RERI, relative excess risk; AP, attributable proportion; SI, synergy index.

Table 6 Measures for estimation of synergic effect between T stage and M stage for the cancer specific survival of DTC

\begin{tabular}{|c|c|c|c|c|c|}
\hline T stage & M stage & Death events (\%) & Total case & $\mathrm{HR}(95 \% \mathrm{Cl})$ & $P$ value \\
\hline T1-T2 & M1 & $34(13.2)$ & 258 & 39.055 (26.034-58.588) & $<0.001^{\star}$ \\
\hline T3-T4 & MO & $377(2.0)$ & 18,662 & 4.756 (3.409-6.636) & $<0.001^{*}$ \\
\hline T3-T4 & M1 & $166(26.9)$ & 616 & 32.505 (22.260-47.465) & $<0.001^{*}$ \\
\hline AP & \multicolumn{5}{|c|}{$0.537(0.367-0.707)$} \\
\hline SI & \multicolumn{5}{|c|}{$2.168(1.498-3.137)$} \\
\hline
\end{tabular}

Adjusted for age at diagnosis, year at diagnosis, sex, race, $\mathrm{N}$ stage, multifocality, histology subtype, extrathyroidal extension, radiation, surgery. ${ }^{*}$, represent the $\mathrm{P}$ value $<0.05$. DTC, differentiated thyroid cancer; RERI, relative excess risk; AP, attributable proportion; SI, synergy index. 
A

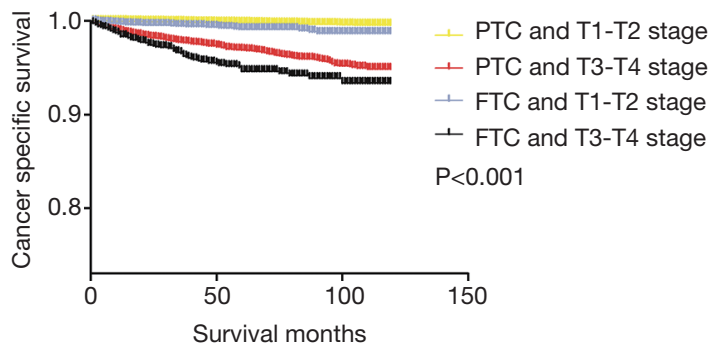

B

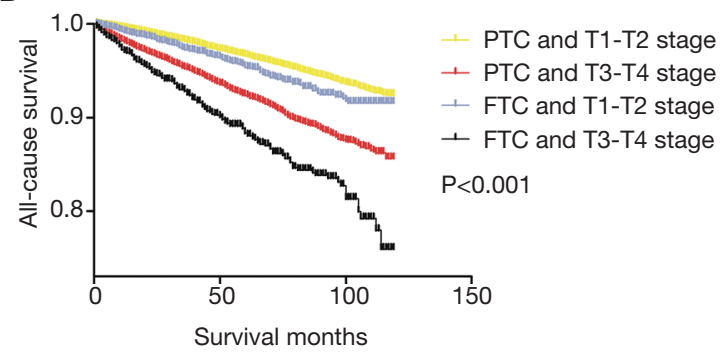

Figure 2 Effects of histology subtype and T stage on: (A) cancer specific survival of patients with DTC; (B) overall survival of patients. DTC, differentiated thyroid cancer; PTC, papillary thyroid cancer; FTC, follicular thyroid cancer.
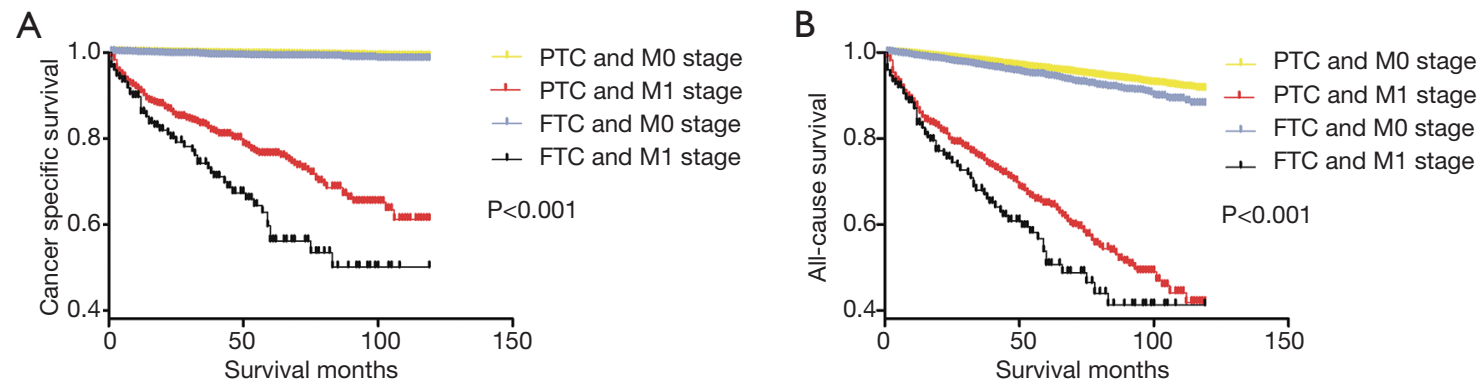

Figure 3 Effects of histology subtype and M stage on: (A) cancer specific survival of patients with DTC; (B) overall survival of patients. DTC, differentiated thyroid cancer; PTC, papillary thyroid cancer; FTC, follicular thyroid cancer.

these results, the RERI was 134.125 , with a $95 \%$ CI ranging from 70.463 to 197.787 , indicating that there was a strong synergistic interaction between T-stage and M-stage on the presence of death. Furthermore, there would be a 134.125fold relative excess risk contributed by the synergistic interaction. The $\mathrm{AP}$ was 0.537 with a $95 \% \mathrm{CI}$ ranging from 0.367 to 0.707 , suggesting that $53.7 \%$ of the deaths caused by exposure to these two risk factors were due to synergistic interactions between $\mathrm{T}$ stage and $\mathrm{M}$ stage. The SI was 2.168 (95\% CI: 1.498-3.137). The synergistic effect between histology subtype and $\mathrm{T}$ stage, histology subtype and $\mathrm{M}$ stage, T stage and $M$ stage for the all-cause survival of DTC were shown in Tables S3-S5.

\section{Kaplan-Meier analysis for survival in DTCs}

Kaplan-Meier survival analysis was performed for four groups divided by histologic subtype and $\mathrm{T}$ stage (Figure 2A). The cancer-specific survival curve of patients with T1-T2 PTC was relatively flat, and other groups were associated with a modest decline in the survival curve. By contrast, the curves for T3-T4 FTC and PTC showed a sharp decline when OS was analyzed (Figure 2B).

Similarly, according to the Kaplan-Meier analysis of four groups divided by histologic subtype and $M$ stage, the cancer-specific survival curve and OS curve of M1 stage FTC and PTC showed a remarkable decline in survival as compared to those of M0 DTC patients (Figure $3 A, B$ ).

The Kaplan-Meier analysis of DTC patients divided into four groups based on T stage and $M$ stage showed that the cancer-specific survival and OS curves for patients with T3T4 plus M1 disease and T1-T2 plus M1 disease showed a significant decline compared to those for patients with M0 disease (Figures $4 A, B$ ).

\section{Discussion}

Histologic subtype, $\mathrm{T}$ stage, and $\mathrm{M}$ stage are reported to be risk factors for death in DTC patients $(10,12-14)$. However, the synergistic effects of these clinicopathological risk factors have not been investigated. In this study, based on data from the SEER database, we extensively explored the value of prognosis of these three factors in patients with DTC, using univariate and multivariate analyses. We 
A

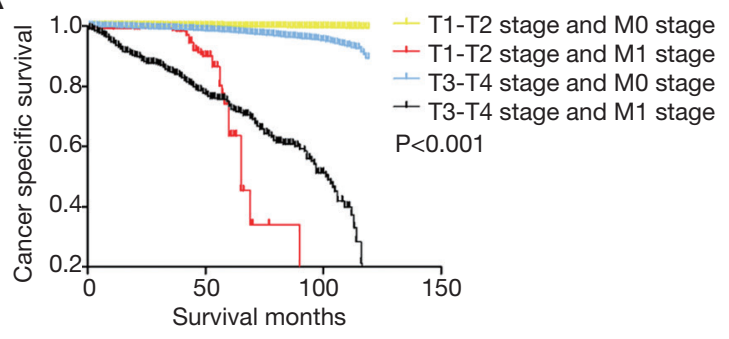

B

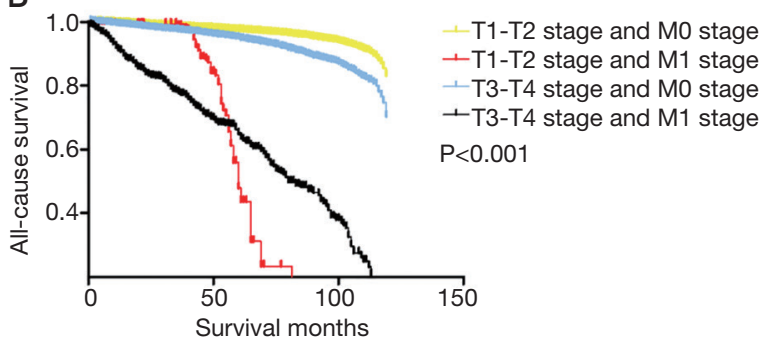

Figure 4 Effects of $T$ stage and $M$ stage on: (A) cancer specific survival of patients with DTC; (B) overall survival of patients with DTC. DTC, differentiated thyroid cancer.

also showed that they are risk factors for CSM and ACM. Next, patients were divided into subgroups according to tumor subtype, $T$ stage, and $M$ stage. We found that within each subgroup, these factors had synergistic effects on the prognosis of DTC. The synergistic effects of histologic subtypes, $T$ and $M$ stage were evaluated by RERI, AP and SI. Based on our results, DTC patients with two out of the three factors (FTC, higher T stage, and metastatic disease) were associated with a higher risk of CSM compared to those who had one or none of the risk factors.

Previous reports have provided decisive information regarding whether FTC has more aggressive clinical features than PTC $(15,16)$. Invasive clinical features, including distant metastasis, are usually associated with shorter cancer-specific survival. In the present study, FTC was associated with higher CSM and ACM rates than PTC. Although angioinvasion was not assessed, it has been reported that vascular invasion has no adverse influence on short-term outcomes or long-term prognosis in FTC or short-term outcomes in PTC (17).

The multi-stage system risk classification of DTC, including assessment of tumor size, is helpful to predict the risk of local recurrence, metastasis, and mortality in DTC patients (14). A previous report suggested that, for small thyroid nodules, a tumor size of $1.5 \mathrm{~cm}$ or larger is not associated with death from thyroid cancer (18). Nguyen et al. have suggested that the probability of distant metastasis of DTC tumors smaller than $4 \mathrm{~cm}$ (lower than T2 stage) is less than $3 \%$, but large tumors (T4 stage) increased the ACM rate (19). In our study, the HRs for CSM of T2, T3, and T4 disease were all higher than that for T1 disease, indicating that the classification of tumor size as a risk stratification factor for the prognosis of thyroid cancer is reasonable. However, Cheema et al. suggested that the size of tumors was not related to the higher recurrence rate, and rather the presence of metastasis is a better predictor of recurrence and prognosis at the time of diagnosis (20). Distant metastasis is relatively rare in DTC; it was observed in only $1.0 \%$ of DTC patients in our study. Multiple metastases of thyroid cancer are associated with a high mortality rate, reaching $92 \%$ at 5 years, so early diagnosis and treatment are necessary (21).

Pathological classification of tumors requires accurate histopathological examination of the specimen after thyroidectomy and lymphadenectomy. T stage and $M$ stage can be assessed by imaging, but ultimately need to be evaluated after surgery. However, they do have a significant impact on the therapeutic options, including surgery, radioactive iodine, and observation. Thyroidectomy is recommended in the following cases: FTC with extensive or vascular invasion, and PTC with tumor size $>1 \mathrm{~cm}$ or with metastatic disease, irrespective of size (14). Adjuvant radioiodine therapy is considered the most effective treatment for non-resectable or incompletely resected DTC (14), or with distant metastasis (22). According to our results, patients with FTC, higher T stage, and M1 stage should be recommended for aggressive treatment.

Neri et al. reported on 191 cases of breast cancer and found multifocal breast cancer to be related to significantly worse prognosis with breast cancer-specific survival (23). In this study, the effect of "multifocality" on cancer-specific survival showed an opposite result between univariate and multivariate analysis. We consider that $\mathrm{P}>0.05$ and $\mathrm{HR}=1.044$ in univariate analysis indicating that the risk of cancer death is not significantly aggravated by single focus. So it is consistent with the results of multivariate analysis which shows that multifocality, not single focus, increased the risk of CSM. We also note that, in Figure 3, DTC with T1-T2 and M1 stage showed a worse cancerspecific survival and all-cause survival than those with 
T3-T4 and M1 stage. We consider it may be that the difference between the two curves is not statistically significant or that there are some confounding factors that influence the results.

The present study has some limitations. First, there may be selection bias, because this is a retrospective study. Second, the patients' family history, vascular invasion status, accurate extension information, and other histological characteristics, which could minimize selection bias, were not taken into account. Finally, detailed data on complications and disease recurrence are not included in the SEER database, so we could not account for them in our analyses.

\section{Conclusions}

This large, comprehensive study, complemented by the SEER data analyses, provides conclusive evidence for the value of histological subtypes, $T$ stage and $M$ stage in the clinical prognosis of DTC. In conclusion, the different combinations of these three factors have synergic effects on the risk of mortality in DTC. This study is likely to have a widespread impact on the current clinical management of DTC.

\section{Acknowledgments}

Funding: None.

\section{Footnote}

Reporting Checklist: The authors have completed the STROBE reporting checklist. Available at http://dx.doi. org/10.21037/tcr-20-359

Peer Review File: Available at http://dx.doi.org/10.21037/tcr20-359

Conflicts of Interest: All authors have completed the ICMJE uniform disclosure form (available at http://dx.doi. org/10.21037/tcr-20-359). The authors have no conflicts of interest to declare.

Ethical Statement: The authors are accountable for all aspects of the work in ensuring that questions related to the accuracy or integrity of any part of the work are appropriately investigated and resolved. The research was conducted in accordance with the Declaration of Helsinki (as revised in 2013). Ethical approval was not required since SEER is a publicly available database with anonymized data.

Open Access Statement: This is an Open Access article distributed in accordance with the Creative Commons Attribution-NonCommercial-NoDerivs 4.0 International License (CC BY-NC-ND 4.0), which permits the noncommercial replication and distribution of the article with the strict proviso that no changes or edits are made and the original work is properly cited (including links to both the formal publication through the relevant DOI and the license). See: https://creativecommons.org/licenses/by-nc-nd/4.0/.

\section{References}

1. Siegel RL, Miller KD, Jemal A. Cancer statistics, 2018. CA Cancer J Clin 2018;68:7-30.

2. Lim H, Devesa SS, Sosa JA, et al. Trends in Thyroid Cancer Incidence and Mortality in the United States, 1974-2013. JAMA 2017;317:1338-48.

3. Kitahara CM, Sosa JA. The changing incidence of thyroid cancer. Nat Rev Endocrinol 2016;12:646-53.

4. Mao Y, Xing M. Recent incidences and differential trends of thyroid cancer in the USA. Endocr Relat Cancer 2016;23:313-22.

5. Murthy SP, Balasubramanian D, Anand A, et al. Extent of Thyroidectomy in Differentiated Thyroid Cancers-Review of Evidence. Indian J Surg Oncol 2018;9:90-6.

6. Shah BR, Griffiths R, Hall SF. Thyroid cancer incidence among Asian immigrants to Ontario, Canada: A population-based cohort study. Cancer 2017;123:3320-5.

7. Noone AM, Cronin KA, Altekruse SF, et al. Cancer Incidence and Survival Trends by Subtype Using Data from the Surveillance Epidemiology and End Results Program, 1992-2013. Cancer Epidemiol Biomarkers Prev 2017;26:632-41.

8. Aboelnaga EM, Ahmed RA. Difference between papillary and follicular thyroid carcinoma outcomes: an experience from Egyptian institution. Cancer Biol Med 2015;12:53-9.

9. Krajewska J, Chmielik E, Jarzab B. Dynamic risk stratification in the follow-up of thyroid cancer: what is still to be discovered in 2017? Endocr Relat Cancer 2017;24:R387-R402.

10. Glikson E, Alon E, Bedrin L, et al. Prognostic Factors in Differentiated Thyroid Cancer Revisited. Isr Med Assoc J 2017;19:114-8. 
11. Qu HJ, Qu XY, Hu Z, et al. The synergic effect of BRAF(V600E) mutation and multifocality on central lymph node metastasis in unilateral papillary thyroid carcinoma. Endocr J 2018;65:113-20.

12. Balachandar S, La Quaglia M, Tuttle RM, et al. Pediatric Differentiated Thyroid Carcinoma of Follicular Cell Origin: Prognostic Significance of Histologic Subtypes. Thyroid 2016;26:219-26.

13. Davies L, Welch HG. Current thyroid cancer trends in the United States. JAMA Otolaryngol Head Neck Surg 2014;140:317-22.

14. Schmidbauer B, Menhart K, Hellwig D, et al.

Differentiated Thyroid Cancer-Treatment: State of the Art. Int J Mol Sci 2017;18:1292.

15. Xu B, Ghossein R. Evolution of the histologic classification of thyroid neoplasms and its impact on clinical management. Eur J Surg Oncol 2018;44:338-47.

16. Nixon IJ, Whitcher MM, Palmer FL, et al. The impact of distant metastases at presentation on prognosis in patients with differentiated carcinoma of the thyroid gland. Thyroid 2012;22:884-9.

17. Furlan JC, Bedard YC, Rosen IB. Clinicopathologic significance of histologic vascular invasion in papillary and follicular thyroid carcinomas. J Am Coll Surg

Cite this article as: Zhou L, Wang S, Chen S, Huang Y, Hu D, Wei W, Zhang C, Wang M, Zhou W, Zeng W, Liu Z, Guo L. Synergistic effects of histologic subtype, T-stage, and M-stage in the prognosis of differentiated thyroid cancer: a retrospective observational study. Transl Cancer Res 2020;9(8):4676-4685. doi: 10.21037/tcr-20-359
2004;198:341-8.

18. Han K, Kim EK, Kwak JY. 1.5-2 cm tumor size was not associated with distant metastasis and mortality in small thyroid cancer: A population-based study. Sci Rep 2017;7:46298.

19. Nguyen XV, Roy Choudhury K, Tessler FN, et al. Effect of Tumor Size on Risk of Metastatic Disease and Survival for Thyroid Cancer: Implications for Biopsy Guidelines. Thyroid 2018;28:295-300.

20. Cheema Y, Repplinger D, Elson D, et al. Is tumor size the best predictor of outcome for papillary thyroid cancer? Ann Surg Oncol 2006;13:1524-8.

21. Rao USV, Koya S, Chatterjee S. Evaluation of Distant Metastasis in Treated Thyroid Cancers: Study of Radioiodine Scans in a Tertiary Care Center. Journal of Head \& Neck Physicians and Surgeons 2016;4:59-62

22. Kreissl M, Janssen M, Nagarajah J. Current treatment strategies in metastasized differentiated thyroid cancer. J Nucl Med 2019;60:9-15.

23. Neri A, Marrelli D, Megha T, et al. Clinical significance of multifocal and multicentric breast cancers and choice of surgical treatment: a retrospective study on a series of 1158 cases. BMC Surg 2015;15:1. 
Table S1 Clinicopathological parameters associated with the cancer-specific survival

\begin{tabular}{|c|c|c|c|c|c|c|c|c|c|}
\hline \multirow{2}{*}{ Parameters } & \multirow{2}{*}{ Subgroup } & \multicolumn{4}{|c|}{ Univariate } & \multicolumn{4}{|c|}{ Multivariate } \\
\hline & & HR & 95\% Cl (lower) & 95\% Cl (upper) & $P$ value & $\mathrm{HR}$ & 95\% Cl (lower) & $95 \% \mathrm{Cl}$ (upper) & $P$ value \\
\hline \multirow[t]{2}{*}{ Age of diagnosis } & $<55$ & Ref & & & & Ref & & & \\
\hline & $\geq 55$ & 8.468 & 6.964 & 10.297 & $<0.001^{*}$ & 1.066 & 1.060 & 1.072 & $<0.001^{*}$ \\
\hline \multirow[t]{2}{*}{ Year at diagnosis } & 2004-2008 & Ref & & & & Ref & & & \\
\hline & 2009-2013 & 0.790 & 0.664 & 0.941 & $0.008^{\star}$ & 0.858 & 0.721 & 1.021 & 0.084 \\
\hline \multirow[t]{2}{*}{ Sex } & Female & Ref & & & & Ref & & & \\
\hline & Male & 2.995 & 2.575 & 3.484 & $<0.001^{*}$ & 1.410 & 1.205 & 1.649 & $<0.001^{*}$ \\
\hline \multirow[t]{3}{*}{ Race } & White & Ref & & & & Ref & & & \\
\hline & Black & 0.806 & 0.567 & 1.145 & 0.228 & 1.024 & 0.716 & 1.465 & 0.895 \\
\hline & Other & 1.381 & 1.110 & 1.719 & $0.004^{*}$ & 0.956 & 0.766 & 1.192 & 0.688 \\
\hline \multirow[t]{4}{*}{ T-stage } & $\mathrm{T} 1$ & Ref & & & & Ref & & & \\
\hline & $\mathrm{T} 2$ & 2.666 & 1.890 & 3.759 & $<0.001^{*}$ & 2.423 & 1.707 & 3.440 & $<0.001^{*}$ \\
\hline & T3 & 8.638 & 6.615 & 11.280 & $<0.001^{*}$ & 4.474 & 3.138 & 6.379 & $<0.001^{*}$ \\
\hline & $\mathrm{T} 4$ & 83.701 & 65.110 & 107.599 & $<0.001^{*}$ & 17.005 & 11.403 & 25.361 & $<0.001^{*}$ \\
\hline \multirow[t]{2}{*}{$\mathrm{N}$-stage } & No & Ref & & & & Ref & & & \\
\hline & $\mathrm{N} 1$ & 4.779 & 4.105 & 5.563 & $<0.001^{*}$ & 1.910 & 1.591 & 2.293 & $<0.001^{*}$ \\
\hline \multirow[t]{2}{*}{ M-stage } & Mo & Ref & & & & Ref & & & \\
\hline & M1 & 48.518 & 41.115 & 57.253 & $<0.001^{*}$ & 6.403 & 5.320 & 7.707 & $<0.001^{*}$ \\
\hline \multirow[t]{2}{*}{ Multifocality } & Yes & Ref & & & & Ref & & & \\
\hline & No & 1.044 & 0.896 & 1.217 & 0.582 & 0.793 & 0.677 & 0.929 & $0.004^{*}$ \\
\hline \multirow[t]{2}{*}{ Histological types } & Papillary & Ref & & & & Ref & & & \\
\hline & Follicular & 2.620 & 2.111 & 3.252 & $<0.001^{*}$ & 1.811 & 1.423 & 2.305 & $<0.001^{*}$ \\
\hline \multirow[t]{2}{*}{ Extrathyroidal extension } & No & Ref & & & & Ref & & & \\
\hline & Yes & 13.363 & 11.308 & 15.791 & $<0.001^{*}$ & 1.388 & 1.024 & 1.883 & $0.035^{\star}$ \\
\hline \multirow[t]{3}{*}{ Radiation } & None or refused & Ref & & & & Ref & & & \\
\hline & $\begin{array}{l}\text { Radiation beam or radioactive } \\
\text { implants }\end{array}$ & 19.730 & 15.905 & 24.473 & $<0.001^{*}$ & 2.953 & 2.337 & 3.732 & $<0.001^{*}$ \\
\hline & $\begin{array}{l}\text { Radioisotopes or radiation } \\
\text { beam plus isotopes or implants }\end{array}$ & 1.592 & 1.334 & 1.899 & $<0.001^{*}$ & 0.785 & 0.651 & 0.948 & $0.012^{*}$ \\
\hline \multirow[t]{3}{*}{ Surgery } & Lobectomy & Ref & & & & Ref & & & \\
\hline & $\begin{array}{l}\text { Subtotal or near total } \\
\text { thyroidectomy }\end{array}$ & 2.086 & 1.393 & 3.124 & $<0.001^{*}$ & 1.319 & 0.875 & 1.988 & 0.186 \\
\hline & Total thyroidectomy & 1.675 & 1.289 & 2.177 & $<0.001^{*}$ & 1.105 & 0.840 & 1.454 & 0.476 \\
\hline
\end{tabular}

${ }^{*}$, represent the $\mathrm{P}$ value $<0.05$. 
Table S2 Clinicopathological parameters associated with the all-cause survival

\begin{tabular}{|c|c|c|c|c|c|c|c|c|c|}
\hline \multirow{2}{*}{ Parameters } & \multirow{2}{*}{ Subgroup } & \multicolumn{4}{|c|}{ Univariate } & \multicolumn{4}{|c|}{ Multivariate } \\
\hline & & $\mathrm{HR}$ & 95\% Cl (lower) & 95\% Cl (upper) & $P$ value & $\mathrm{HR}$ & 95\% Cl (lower) & $95 \% \mathrm{Cl}$ (upper) & $P$ value \\
\hline \multirow[t]{2}{*}{ Age of diagnosis } & $<55$ & Ref & & & & Ref & & & \\
\hline & $\geq 55$ & 6.848 & 6.313 & 7.428 & $<0.001^{*}$ & 5.794 & 5.331 & 6.296 & $<0.001^{*}$ \\
\hline \multirow[t]{2}{*}{ Year at diagnosis } & 2004-2008 & Ref & & & & Ref & & & \\
\hline & 2009-2013 & 0.949 & 0.873 & 1.031 & 0.215 & 0.929 & 0.855 & 1.009 & 0.082 \\
\hline \multirow[t]{2}{*}{ Sex } & Female & Ref & & & & Ref & & & \\
\hline & Male & 2.395 & 2.237 & 2.565 & $<0.001^{*}$ & 1.669 & 1.555 & 1.792 & $<0.001^{*}$ \\
\hline \multirow[t]{3}{*}{ Race } & White & Ref & & & & Ref & & & \\
\hline & Black & 1.229 & 1.082 & 1.396 & $0.002^{*}$ & 1.276 & 1.122 & 1.452 & $<0.001^{*}$ \\
\hline & Other & 0.844 & 0.748 & 0.952 & $0.006^{*}$ & 0.795 & 0.704 & 0.897 & $<0.001^{*}$ \\
\hline \multirow[t]{4}{*}{ T-stage } & $\mathrm{T} 1$ & Ref & & & & Ref & & & \\
\hline & T2 & 1.013 & 0.914 & 1.123 & 0.800 & 1.136 & 1.020 & 1.264 & $0.020^{*}$ \\
\hline & T3 & 1.576 & 1.446 & 1.718 & $<0.001^{*}$ & 1.353 & 1.181 & 1.550 & $<0.001^{*}$ \\
\hline & T4 & 6.531 & 5.935 & 7.186 & $<0.001^{*}$ & 3.414 & 2.852 & 4.088 & $<0.001^{*}$ \\
\hline \multirow[t]{2}{*}{$\mathrm{N}$-stage } & No & Ref & & & & Ref & & & \\
\hline & N1 & 1.510 & 1.401 & 1.628 & $<0.001^{*}$ & 1.374 & 1.255 & 1.503 & $<0.001^{*}$ \\
\hline \multirow[t]{2}{*}{ M-stage } & MO & Ref & & & & Ref & & & \\
\hline & M1 & 11.590 & 10.286 & 13.059 & $<0.001^{*}$ & 4.069 & 3.568 & 4.641 & $<0.001^{*}$ \\
\hline \multirow[t]{2}{*}{ Multifocality } & Yes & Ref & & & & Ref & & & \\
\hline & No & 0.977 & 0.912 & 1.046 & 0.503 & 0.951 & 0.885 & 1.022 & 0.173 \\
\hline \multirow[t]{2}{*}{ Histological types } & Papillary & Ref & & & & Ref & & & \\
\hline & Follicular & 1.630 & 1.454 & 1.829 & $<0.001^{*}$ & 1.327 & 1.170 & 1.505 & $<0.001^{*}$ \\
\hline \multirow[t]{2}{*}{ Extrathyroidal extension } & No & Ref & & & & Ref & & & \\
\hline & Yes & 2.466 & 2.294 & 2.652 & $<0.001^{*}$ & 1.097 & 0.943 & 1.277 & 0.228 \\
\hline \multirow[t]{3}{*}{ Radiation } & None or refused & Ref & & & & Ref & & & \\
\hline & Radiation beam or radioactive implants & 3.188 & 2.765 & 3.675 & $<0.001^{*}$ & 1.492 & 1.281 & 1.737 & $<0.001^{*}$ \\
\hline & $\begin{array}{l}\text { Radioisotopes or radiation beam plus } \\
\text { isotopes or implants }\end{array}$ & 0.746 & 0.696 & 0.800 & $<0.001^{*}$ & 0.642 & 0.595 & .694 & $<0.001^{*}$ \\
\hline \multirow[t]{3}{*}{ Surgery } & Lobectomy & Ref & & & & Ref & & & \\
\hline & Subtotal or near total thyroidectomy & 1.053 & 0.890 & 1.245 & 0.548 & 1.035 & 0.874 & 1.226 & 0.692 \\
\hline & Total thyroidectomy & 0.865 & 0.789 & 0.947 & $0.002^{*}$ & 0.918 & 0.832 & 1.012 & 0.084 \\
\hline
\end{tabular}

*, represent the $P$ value $<0.05$. 
Table S3 Measures for estimation of synergic effect between histology subtype and T stage for the all-cause survival of DTC

\begin{tabular}{|c|c|c|c|c|c|}
\hline Histology subtype & T stage & Death events (\%) & Total case & $\mathrm{HR}(95 \% \mathrm{Cl})$ & $P$ value \\
\hline PTC & T3-T4 & $1,156(6.6)$ & 17,612 & $1.251(1.083-1.445)$ & $0.002^{*}$ \\
\hline FTC & T1-T2 & $139(4.4)$ & 3,144 & $1.226(1.030-1.459)$ & $0.022^{*}$ \\
\hline FTC & T3-T4 & $184(11.0)$ & 1666 & 1.919 (1.623-2.268) & $<0.001^{*}$ \\
\hline AP & \multicolumn{5}{|c|}{$0.311(0.183-0.439)$} \\
\hline SI & \multicolumn{5}{|c|}{$1.706(1.310-2.220)$} \\
\hline
\end{tabular}

Adjusted for age at diagnosis, year at diagnosis, sex, race, N stage, M stage, multifocality, extrathyroidal extension, radiation, surgery. *, represent the $\mathrm{P}$ value $<0.05$. DTC, differentiated thyroid cancer; PTC, papillary thyroid cancer; FTC, follicular thyroid cancer; RERI, relative excess risk; AP, attributable proportion; SI, synergy index.

Table S4 Measures for estimation of synergic effect between histology subtype and M stage for the all-cause survival of DTC

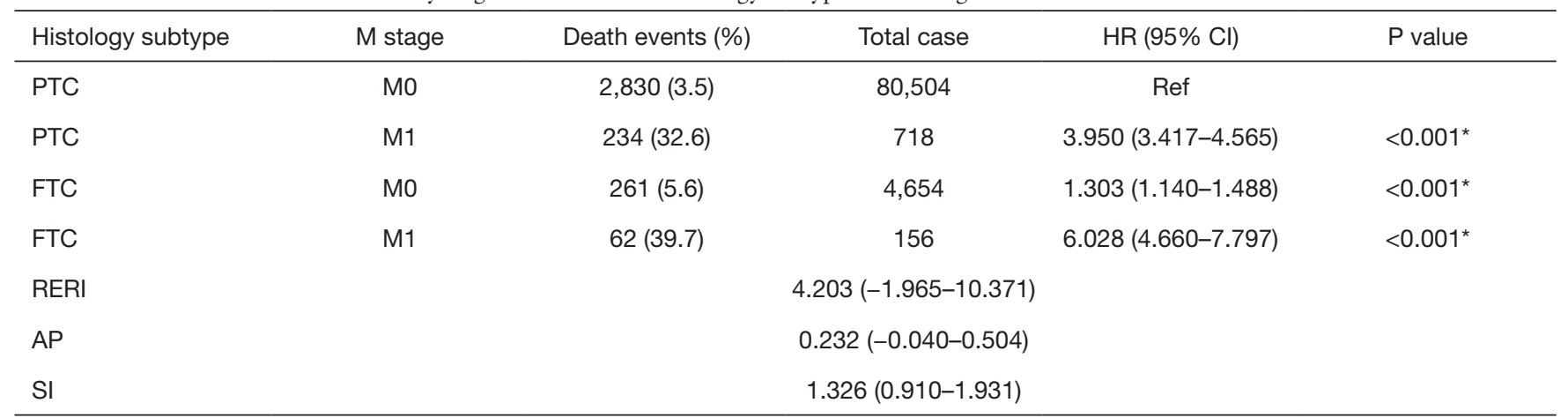

Adjusted for age at diagnosis, year at diagnosis, sex, race, T stage, $\mathrm{N}$ stage, multifocality, extrathyroidal extension, radiation, surgery. *, represent the $\mathrm{P}$ value $<0.05$. DTC, differentiated thyroid cancer; PTC, papillary thyroid cancer; FTC, follicular thyroid cancer; RERI, relative excess risk; AP, attributable proportion; SI, synergy index.

Table S5 Measures for estimation of synergic effect between T stage and M stage for the all-cause survival of DTC

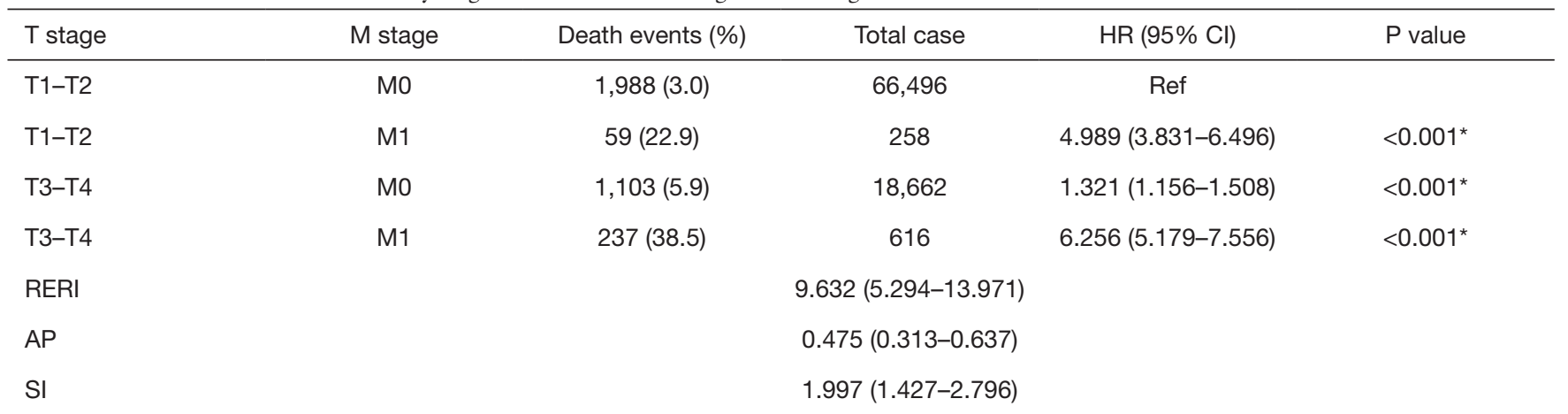

Adjusted for age at diagnosis, year at diagnosis, sex, race, $\mathrm{N}$ stage, multifocality, histology subtype, extrathyroidal extension, radiation, surgery. *, represent the P value $<0.05$. DTC, differentiated thyroid cancer; RERI, relative excess risk; AP, attributable proportion; SI, synergy index. 